\title{
Shared Environment Effects on Children's Emotion Recognition
}

\author{
Rotem Schapira ${ }^{1,2 *}$, Hillary Anger Elfenbein ${ }^{3}$, Meirav Amichay-Setter ${ }^{4}$, \\ Carolyn Zahn-Waxler ${ }^{5}$ and Ariel Knafo-Noam ${ }^{4}$ \\ ${ }^{1}$ The Mofet Institute, Tel-Aviv, Israel, ${ }^{2}$ Levinsky College of Education, Tel-Aviv, Israel, ${ }^{3}$ Washington University, St. Louis, MI, \\ United States, ${ }^{4}$ The Hebrew University of Jerusalem, Jerusalem, Israel, ${ }^{5}$ University of Wisconsin-Madison, Madison, WI, \\ United States
}

Empathy is relevant to many psychiatric conditions. Empathy involves the natural ability to perceive and be sensitive to the emotional states of others. Thus, emotion recognition (ER) abilities are key to understanding empathy. Despite the importance of ER to normal and abnormal social interactions, little is known about how it develops throughout childhood. We examined genetic and environmental influences on children's ER via facial and vocal cues in 344 7-year-old twin children [59 monozygotic (MZ) and 113 same-sex dizygotic (DZ) pairs], who were part of the Longitudinal Israeli Study of Twins. ER was assessed with

\section{OPEN ACCESS}

Edited by:

Simon Surguladze,

King's College London,

United Kingdom

Reviewed by:

Nils Kohn,

Radboud University

Netherlands

Hedwig Eisenbarth,

Victoria University of Wellington,

New Zealand

*Correspondence:

Rotem Schapira

rotemschapira@gmail.com

Specialty section: This article was submitted to

Social Cognition,

a section of the journa

Frontiers in Psychiatry

Received: 09 January 2019 Accepted: 25 March 2019

Published: 11 April 2019

Citation:

Schapira R, Anger Elfenbein $\mathrm{H}$, Amichay-Setter M, Zahn-Waxler C

and Knafo-Noam A (2019)

Shared Environment Effects on

Children's Emotion Recognition.

Front. Psychiatry 10:215.

doi: 10.3389/fpsyt.2019.00215 the child version of the Diagnostic Assessment of Nonverbal Accuracy. For both facial and vocal cues of emotion, twin correlations were not higher for MZ twins than for DZ twins, suggesting no heritability for ER in this population. In contrast, correlations were positive for both types of twins, indicating a shared environmental effect. This was supported by a bivariate genetic analysis. This pattern was robust to controlling for twins being of the same sex and age. Effects remained after controlling for background variables such as family income and number of additional siblings. The analysis found a shared environmental correlation between facial and vocal ER $\left(r_{c}=.63\right)$, indicating that the shared environmental factors contributed to the overlap between vocal and facial ER. The study highlights the importance of the shared environment to children's ER.

Keywords: empathy, emotion recognition, shared environment effect, individual differences, childhood

\section{INTRODUCTION}

Empathy, the ability to perceive and be sensitive to others' emotional states (1), is relevant to many psychiatric conditions (2). Emotion recognition (ER) abilities are relevant to feeling empathy for others (3), specifically cognitive empathy (4), the ability to recognize and understand the emotions of others (5). Despite the importance of ER for social interaction and functioning (6-8), individuals vary markedly in ER ability $(9,10)$. Our research addresses the origin of these individual differences. Specifically, we investigated genetic and environmental effects on children's recognition of emotion from facial and vocal cues using data from seven-year-old twins.

Childhood may be a unique developmental period for ER, with important developmental advances such as the ability to take others' perspective, which contribute to better understanding of emotions and social interactions (11-13). Nevertheless, little is known about how ER develops throughout childhood [e.g., Ref. (12)]. Most of the studies on the contribution of the family context to children's emotional development did not directly address ER but focused on related skills, such as children's emotion understanding (14). Many environmental factors may 
influence social development, including parental socialization, peer influence, teachers, school, and culture (15-17). Much research has also addressed the roles of socioeconomic status (SES) and sex on ER development (18). There is evidence that children with low SES show difficulties in terms of their overall emotional development (19) and, particularly, in ER (20), and in a meta-analysis of 215 studies (21), females had a small but reliable advantage in ER tasks.

Although theoretical and empirical research suggests that individual differences in empathy are affected by both genetic and environmental factors (22), only little research has examined genetic and environmental effects on ER. Studies estimating genetic and environmental influences on empathy have typically relied on the classic twin design, which compares monozygotic (MZ) and dizygotic (DZ) twins (23). Higher similarity between MZ than DZ twins indicates genetic influence (heritability), while twin similarity that is not higher for MZ twins cannot be accounted for by their genetic relatedness and is attributed to the shared environment. Finally, dissimilarity between family members despite their genetic and environmental relatedness indicates the influence of the nonshared environment and measurement error.

We found only two past studies of genetic effects on ER. Studying 10 -year-old twins ( $N=250$ pairs), Lau and colleagues (24) found modest and largely nonsignificant genetic effects on recognition of specific emotions from facial expressions, and a strong (75\% of the variance) genetic effect on a global factor estimated across emotions. The second study (25) also examined facial emotion recognition in a larger sample of twins $(N=957$ individuals) in a wide range of ages, 9 to 17. The findings show a significant genetic effect $(34 \%-57 \%)$ for the recognition of six basic emotions. Only modest evidence was found for shared environment effects (1\%-12\%), controlling for age and sex. Nonshared environmental effects accounted for the remaining variance in both studies.

Substantial changes occur in ER during middle childhood $(11,12)$. For example, age plays an important role in the emotion comprehension process, and cognitive nonverbal factors are predictors of 3- to 10-year-olds' emotion comprehension (26). Moreover, past work has shown that the relative importance of genetic and environmental effects changes with age [for a metaanalysis on empathy, see Ref. (22)]. It is therefore important to extend the results to younger samples. Based on past work, we expected to find a genetic effect and nonshared environmental influence on children's ER in our 7-year-old sample. Additionally, based on the above evidence, we examined the role of SES and sex in ER.

Past work (24) focused on facial expressions. Importantly, recent studies have shown the importance of vocal cues to accurate ER (27). Vocal cues improve nonverbal communication and ER in social situations [e.g., Refs. $(28,29)]$. While preschool children tend to rely more on facial expressions during social interaction, school-aged children (ages 7-12) rely on both facial expression and tone of voice (30). The importance of vocal cues to emotion, then, calls for studying them in addition to facial cues. Our study, therefore, expands the scope of ER by testing both facial expressions and vocal tone. In addition, studying both kinds of cues in the same design enables an investigation of the origin of the association between understanding vocal and facial cues to emotion. Overlapping genetic effects on facial and vocal ER would indicate a global cross-modality genetic tendency. In contrast, overlapping environmental effects will indicate that similar environmental forces promote (or hinder) development of ER across modalities.

\section{METHODS}

\section{Participants}

A total of 344 Jewish Israeli children (52\% male, $59 \mathrm{MZ}$ pairs and 114 same-sex DZ pairs) participated in the Longitudinal Israeli Study of Twins (LIST) (31) at the age of 7 years (90.05 \pm 3.87 months). Children were observed performing a variety of tasks in the lab. Each child was tested separately by a different experimenter from his or her twin to avoid any bias effects. Written informed consent was obtained from the participants' parents.

\section{Measures}

\section{Emotion Recognition Measure}

We used the child version of the Diagnostic Assessment of Nonverbal Accuracy Scale-2 (DANVA-2) (7, 32, 33). Children watched 24 different pictures of children's faces one at a time and classified each as angry, happy, fearful, or sad. Similarly, they are required to identify the different emotions in 24 recordings of oral speech, where the words themselves are emotionally neutral (the same sentence is used: "I am going out of the room now but I will be back later"). The DANVA has been extensively used and well validated in child samples [e.g., Refs. (34-36)]. Inter-item reliability of the items yielded Cronbach's $\alpha=.70$. Facial and vocal cues correlated positively $(r=.30, p<.001)$ and were analyzed separately as well as summed into an overall ER score.

\section{Demographic Data}

Mothers reported demographic data, including number of additional siblings and SES. SES was indexed by family income, asking parents to rate their income relative to the given national average using a scale ranging from 1 "a lot below" to 5 "a lot above" the average $(M=3.26, \mathrm{SD}=1.26)$.

\section{Analyses}

We performed descriptive analyses with SPSS (version 25). Genetic analyses were performed using the Mx structural equation modeling software (37). Mx was specifically designed to analyze twin data, estimating the relative contribution of additive genetic (A), shared environment (C), and nonshared environment and error (E) effects on individual differences. We also used a bivariate extension of the twin design using the correlated factors model (38), which estimated the ACE components for each modality separately, as well as the associations between the genetic and environmental components contributing to each modality. 


\section{RESULTS}

Table 1 presents descriptive statistics and correlations between $\mathrm{MZ}$ and DZ twins for overall ER and for facial and vocal cues separately. Results showed positive correlations in both MZ and DZ twin pairs. Correlations were not higher for MZ twins, indicating no heritability for ER in this population. Instead, positive correlations for both $\mathrm{DZ}$ and $\mathrm{MZ}$ twins indicate that at least part of the individual differences in these measures is associated with shared environmental factors. Although the DZ correlation was somewhat higher than the MZ correlation, this difference was not significant (Fisher's test of independent correlations, $z=-1.85)$. Similar correlation patterns were found for both facial and vocal cues of emotion.

Our first genetic analysis fitted a univariate genetic model to the overall ER scores. As the genetic effects were estimated at zero, they were dropped from the model without affecting model fit $\left[\Delta \chi^{2}(d f=1)=0.00\right]$. Thus, the model without a genetic effect (CE) was preferred over the less parsimonious full model (ACE). The shared environment effect accounted for $44 \%$ of the variance, and the remaining variance was accounted for by the nonshared environment effect and error (Table 1). We estimated genetic and environmental contributions to facial and vocal ER, as well as the association between these two variables. We fitted a bivariate genetic model to the data. Again, genetic effects were estimated at 0 and could be dropped from the model without worsening fit $\left[\Delta \chi^{2}(d f=3)=0.00\right]$, and the more parsimonious CE model was preferred. The shared environment component accounted for $32 \%$ and $38 \%$ of the variance in facial and vocal ER, respectively. The bivariate genetic analysis indicated that the correlation between facial and vocal ER reflected a shared environmental correlation between these variables $\left[r_{\mathrm{c}}=.63,95 \%\right.$ confidence interval $(\mathrm{CI})=$ .34-.94], with little correlation between the nonshared environment components $\left(r_{\mathrm{e}}=.09,95 \% \mathrm{CI}=-.05\right.$ to .25$)$ (Figure 1). The shared environment effect accounted for $73 \%$ of the correlation between facial and vocal ER (based on the product of $r_{\mathrm{c}}$ and the nonsquared standardized shared environment path coefficients) with the rest of the association accounted for by the nonshared environment effect.
Table 1 presents fit indices for the univariate and bivariate $\mathrm{CE}$ models. These model fit indices reflected the pattern in which similarity between the DZ twins is greater than the MZ twins, which is not expected in the CE model given that the shared environment is estimated as affecting siblings growing up together similarly regardless of genetic similarity.

Age variation in months within our sample correlated with facial ER $(r=.23, p<.01)$ and vocal ER $(r=.22, p<.01)$. In addition, girls performed better than boys in facial $[t(376)=$ $-4.43, p<.004, D=.46]$ and vocal ER $[t(375)=-1.72, p<.001$, $D=.18$ ], in line with previous work $(21,30)$. It was therefore important to account for age and sex differences among twin pairs and to verify that the shared environment effects found in the study go beyond the effects of twins sharing their sex and age. We thus calculated a new ER variable, partialing out the effects of sex and age in a regression analysis. Controlling for sex and age, the results still held $(r \mathrm{MZ}=.23, r \mathrm{DZ}=.46)$, showing no genetic effect. That is, age and sex did not inflate twin correlations and could not account for the shared environment effects estimated.

ER correlated modestly with demographic variables such as greater SES $(r=.14, p<.05)$ and fewer additional siblings $(r=-.16, p<.001)$. However, as was the case with age and sex, follow-up analyses showed that the presence of the shared environment effects was beyond twins' sharing these variables. Specifically, analysis of scores residualized for number of siblings, SES, sex, and age did not substantially change the results, including the lack of genetic effect. The shared environment component was estimated at $23 \%(\mathrm{CI}=.07-.38)$ and $38 \%(\mathrm{CI}=$ $.23-.51$ ) of the variance in facial and vocal ER, respectively, and a shared environmental correlation accounted for the association between these variables $\left(r_{\mathrm{c}}=.46, \mathrm{CI}=.06-.88\right)$.

\section{DISCUSSION}

This study examined genetic and environmental influences on children's ER, for the first time adding vocal to facial cues of

TABLE 1 | Twin correlations and genetic/environmental influences on ER.

\begin{tabular}{|c|c|c|c|c|c|c|c|}
\hline & \multicolumn{2}{|c|}{ Correlations } & \multicolumn{3}{|c|}{$\begin{array}{l}\text { Variance component estimates } \\
\text { proportion ( } 95 \% \mathrm{Cls})\end{array}$} & \multicolumn{2}{|c|}{ Model fit indices } \\
\hline & MZ twins & DZ twins & Genetics & $\begin{array}{c}\text { Shared } \\
\text { environment }\end{array}$ & $\begin{array}{l}\text { Nonshared } \\
\text { environment }\end{array}$ & AIC & BIC \\
\hline Total ER & $.35^{\star \star}$ & $.49^{\star \star}$ & $\begin{array}{c}.00(.00-.24) \\
-\end{array}$ & $\begin{array}{c}.44(.24-.56) \\
{[.44(.32-.56)]}\end{array}$ & $\begin{array}{c}.55(.44-.68) \\
{[.56(.44-.68)]}\end{array}$ & $\begin{array}{l}263.99 \\
261.99\end{array}$ & $\begin{array}{l}-396.18 \\
-398.76\end{array}$ \\
\hline$M(\mathrm{SD})$ & 24.32 (6.12) & $25.48(5.85)$ & & & & & \\
\hline Facial & $.29^{\star \star}$ & $.35^{\star \star}$ & $\begin{array}{c}.00(.00-.38) \\
-\end{array}$ & $\begin{array}{l}.32(.03-.45) \\
{[.32(.18-.45)]}\end{array}$ & $\begin{array}{l}.68(.52-.82) \\
{[.68(.55-.82)]}\end{array}$ & $\begin{array}{l}540.72 \\
534.72\end{array}$ & $\begin{array}{l}-781.26 \\
-788.99\end{array}$ \\
\hline$M(\mathrm{SD})$ & $15.48(4.40)$ & $16.41(4.11)$ & & & & & \\
\hline Vocal & .21 & $.47^{\star \star}$ & $\begin{array}{c}.00(.00-.19) \\
-\end{array}$ & $\begin{array}{c}.38(.19-.50) \\
{[.38(.24-.50)]}\end{array}$ & $\begin{array}{c}.63(.50-.76) \\
{[.63(.50-.76)]}\end{array}$ & $\begin{array}{l}540.72 \\
534.72\end{array}$ & $\begin{array}{l}-781.26 \\
-788.99\end{array}$ \\
\hline$M(\mathrm{SD})$ & $8.84(3.37)$ & $9.03(3.02)$ & & & & & \\
\hline
\end{tabular}

The second line for each variable represents the best-fitting model after dropping the genetic effect estimated at 0.00 . The parentheses portray the estimates from the full ACE model including the genetics, whereas the brackets contain the estimates from the model without the genetics. AlC, Akaike information criterion, calculated in comparison to the full ACE model. BIC, Bayesian information criterion. Cl, confidence intervals. MZ, monozygotic, DZ, dizygotic, same-sex twins. ${ }^{*} p<.05$, ${ }^{* *} p<.001$ 


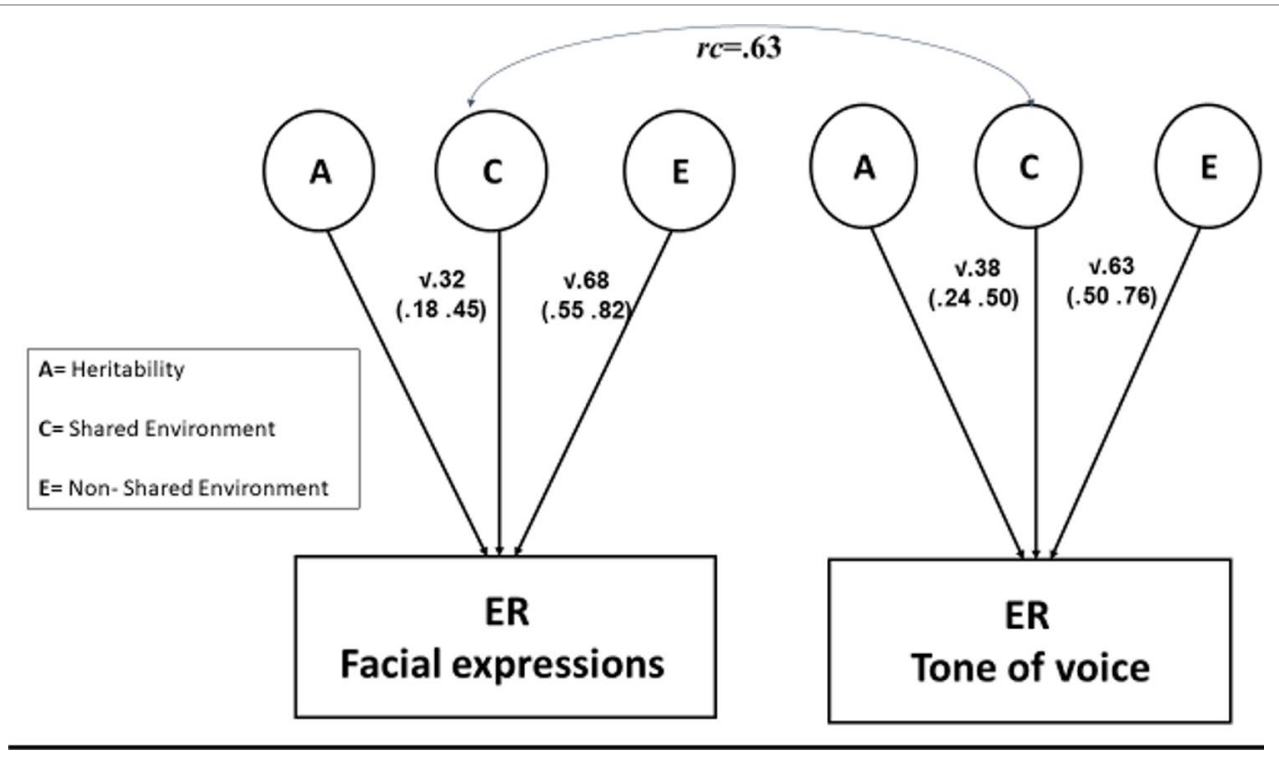

FIGURE 1 | The bivariate model: genetic and environmental influences on ER.

emotion. Our results indicate that individual differences in ER by 7-year-old children are accounted for by shared and nonshared environmental variables. Moreover, the association between facial and vocal cues reflected mainly overlapping shared environmental effects. This study highlights the importance of the environment to children's ER.

Shared environment effects suggest that the family milieu plays an important role in the development of children's ER (16), although the exact process needs further research. Moreover, the bivariate analysis indicated that shared environmental factors largely account for the association between vocal and facial ER. Across species, the social environment provides a place for training and learning about the emotional world, helped by social factors such as contact and familiarity (39). Research has shown that it is easier for individuals to identify others' emotion expressions from their own cultural in-group $(40,41)$. In addition, culture may be influential through stereotypical displays found in various media (42). These archetypes provide an opportunity to gain exposure and learn about the emotional social world. Going to the family level, it is possible that differences among families in the expression of emotions affect children. These shared experiences may affect the ability to understand emotions in a stereotypical and nonexhaustive way, increasing similarity between siblings being exposed to similar events in their family.

A large portion of the individual differences in ER in this study were attributed to nonshared environment effects (43). Nonshared effects are child specific and can include life events such as illness and relationships with family and peers. The study focused on 7-year-olds who, in the Israeli context, are already attending school. School may serve as an important source of nonshared environmental influences, as twins are exposed to a variety of different peers and often different classrooms.
Communicating with diverse children exposes children to others' varied emotional states. This can then enable further peer experience and expertise in ER, which may contribute to the nonshared environment effects on ER.

We did not find any genetic effects, while most past twin studies on empathy and related variables found meaningful genetic effects and little evidence for shared environmental effects $(22,36,44,45)$. One possibility is that our use of a test method vs. the more commonly used questionnaire methods led to lower heritability estimates, as was found for other variables (e.g., parenting) (46). However, past work on facial expressions did find genetic effects (25). Another possibility is that the lack of genetic effects reflects the younger age of our sample as compared to past studies $(5,24)$. Indeed, heritability increases with age for several traits $(47,48)$, including empathy (22), in longitudinal studies using the same method across ages (49).

Thus, further longitudinal research might support the increase in heritability with age. One way in which heritability might increase with age is through evocative gene-environment correlation processes, in which the child's genetically influenced traits increasingly affect the environment, which in turn influences the developing person, leading to an increase in heritability with age (50-52). In addition, the absence of heritability should be interpreted in light of the possibility that genetic effects are moderated or influenced by environmental factors, known as gene-environment interactions.

The consistency of results for facial and vocal cues strengthens our findings. Similarly, controlling for the effects of sex and age, the results still held and showed no genetic effect while highlighting the importance of the shared environment. We note that the sample is not large and focused only on 7-year-olds. Future studies should increase 
the sample size and examine different ages and development over time.

We tested children's ER directly using the DANVA $(29,53)$. As the two were examined in separate rooms, they could not affect one another during testing. At the same time, the DANVA emphasizes very specific facial expressions that could be influenced by family background. Future work should also use subtler cues to express emotion in a more complex and less stereotypical way. In addition, in real life, emotion is perceived through an integration of visual and auditory cues (27), and thus, a pathway for future work is to study vocal and facial cues jointly in the same stimulus.

Our findings contribute to understanding the development of emotion recognition, a core aspect of empathy. They call for in-depth investigation of environmental factors involved in psychiatric disorders characterized by difficulties in emotional recognition.

\section{ETHICS STATEMENT}

The study was approved by the Ethics Committee of the Hebrew University of Jerusalem, Israel.

\section{REFERENCES}

1. Decety J, Jackson PL. A social-neuroscience perspective on empathy. Curr Dir Psychol Sci (2006) 15(2):54-8. doi: 10.1111/j.0963-7214.2006.00406.x

2. Blair RJR. Responding to the emotions of others: dissociating forms of empathy through the study of typical and psychiatric populations. Conscious $\operatorname{Cogn}(2005)$ 14(4):698-718. doi: 10.1016/j.concog.2005.06.004

3. Decety J, Hodges SD. The Social Neuroscience of Empathy. U PAM Van Lang \& L. Lawrence Erlbaum Associates, Mahwah, New Yersey, London. (2006) p. 103-10.

4. Shamay-Tsoory SG, Aharon-Peretz J, Perry D. Two systems for empathy: a double dissociation between emotional and cognitive empathy in inferior frontal gyrus versus ventromedial prefrontal lesions. Brain (2009) 132(3):617-27. doi: 10.1093/brain/awn279

5. Uzefovsky F, Knafo-Noam A. Empathy development throughout the lifespan. In: Sommerville J Decety J. Social Cognition: Frontiers in Developmental Science Series. New York, USA: Psychology Press | Taylor and Francis Group (2017).

6. Garner PW, Waajid B. Emotion knowledge and self regulation as predictors of preschoolers' cognitive ability, classroom behavior, and social competence. J Psychoeduc Assess (2012) 30(4):330-43. doi: 10.1177/0734282912449441

7. Nowicki S, Duke MP. Individual differences in the nonverbal communication of affect: the diagnostic analysis of nonverbal accuracy scale. J Nonverbal Behav (1994) 18(1):9-35. doi: 10.1007/BF02169077

8. Rosenthal R, Hall JA, DiMatteo MR, Rogers PL, Archer D. Sensitivity to nonverbal communication: The PONS Test. Baltimore: Johns Hopkins University Press (1979).

9. Hall JA, Bernieri FJ, Carney DR. Nonverbal behavior and interpersonal sensitivity. In: The new handbook of methods in nonverbal behavior research. Oxford: Oxford University Press (2005). p. 237-81.

10. Lewis GJ, Lefevre CE, Young AW. Functional architecture of visual emotion recognition ability: a latent variable approach. J Exp Psychol Gen (2016) 145(5):589. doi: $10.1037 / \mathrm{xge} 0000160$

11. Gao X, Maurer D. A happy story: developmental changes in children's sensitivity to facial expressions of varying intensities. J Exp Child Psychol (2010) 107(2):67-86. doi: 10.1016/j.jecp.2010.05.003

12. Lawrence K, Campbell R, Skuse D. Age, gender, and puberty influence the development of facial emotion recognition. Front Psychol (2015) 6:761. doi: 10.3389/fpsyg.2015.00761

\section{AUTHOR CONTRIBUTIONS}

RS, AK-N, HA, and CZ-W contributed to the conception and design of the study. RS organized the database. AK-N performed the statistical analysis. RS wrote the first draft of the manuscript. AK-N, HA, MA-S, and CZ-W wrote sections of the manuscript. All authors contributed to manuscript revision and read and approved the submitted version.

\section{FUNDING}

This study was conducted with the support of MOFET Institute, the John Templeton Foundation, and Levinsky College of Education in Tel-Aviv, Israel.

\section{ACKNOWLEDGMENTS}

This study was conducted with the support of MOFET Institute and by a grant from The Science of Generosity Initiative, funded by the John Templeton Foundation to Ariel Knafo.

13. Eccles JS. The development of children ages 6 to 14. Future Child (1999) 9(2):30-44. doi: 10.2307/1602703

14. Denham SA, Bassett HH, Wyatt T. The socialization of emotional competence. In: Handbook of socialization: Theory and research. New York, NY, US: Guildford Press (2007). p. 614-37.

15. Berzenski SR, Yates TM. The differential influences of parenting and child narrative coherence on the development of emotion recognition. Dev Psychol (2017) 53(10):1912. doi: 10.1037/dev0000395

16. Castro VL, Halberstadt AG, Lozada FT, Craig AB. Parents' emotion-related beliefs, behaviours, and skills predict children's recognition of emotion. Infant Child Dev (2015) 24(1):1-22. doi: 10.1002/icd.1868

17. Eisenberg N, Fabes RA, Spinrad TL, Knafo-Noam A. Prosocial development In: Eisenberg N, Damon W, Lerner RM Handbook of child psychology, Vol. 3, Social, emotional, and personality development. 6th New York: Wiley (2016). p. 646-718.

18. Herba C, Phillips M. Annotation: development of facial expression recognition from childhood to adolescence: behavioural and neurological perspectives. J Child Psychol Psychiatry (2004) 45(7):1185-98. doi: 10.1111/ j.1469-7610.2004.00316.x

19. Caspi A, Taylor A, Moffitt TE, Plomin R. Neighborhood deprivation affects children's mental health: environmental risks identified in a genetic design. Psychol Sci (2000) 11(4):338-42. doi: 10.1111/1467-9280.00267

20. Hall JA, Halberstadt AG, O'brien CE. "Subordination" and nonverbal sensitivity: a study and synthesis of findings based on trait measures. Sex Roles (1997) 37(5-6):295-317. doi: 10.1023/A:1025608105284

21. Thompson AE, Voyer D. Sex differences in the ability to recognize nonverbal displays of emotion: a meta-analysis. Cogn Emot (2014) 28(7):116495. doi: 10.1080/02699931.2013.875889

22. Knafo A, Uzefovsky FLegerstee M, Haley DW, Bornstein MH. The developing infant mind: Integrating biology and experience. New York: Guilford Press (2013).

23. Plomin R, DeFries JC, McClearn GE, McGuffin P. Behavioral genetics. 4th. New York: Worth (2001).

24. Lau JY, Burt M, Leibenluft E, Pine DS, Rijsdijk F, Shiffrin N, et al. Individual differences in children's facial expression recognition ability: the role of nature and nurture. Dev Neuropsychol (2009) 34(1):37-51. doi: 10.1080/87565640802564424

25. Rappaport LM, Carney DM, Verhulst B, Neale MC, Blair J, Brotman MA, et al. A developmental twin study of emotion recognition and its negative 
affective clinical correlates. J Am Acad Child Adolesc (2018) 57(12):925-33. doi: 10.1016/j.jaac.2018.05.028

26. Albanese O, De Stasio S, Di Chiacchio C, Fiorilli C, Pons F. Emotion comprehension: the impact of nonverbal intelligence. J Genet Psychol (2010) 171(2):101-15. doi: 10.1080/00221320903548084

27. Atias D, Todorov A, Liraz S, Eidinger A, Dror I, Maymon Y, et al. Loud and unclear: intense real-life vocalizations during affective situations are perceptually ambiguous and contextually malleable. J Exp Psychol Gen (2018) 1-12. doi: 10.1037/xge0000535

28. Kraus MW. Voice-only communication enhances empathic accuracy. Am Psychol (2017) 72(7):644. doi: 10.1037/amp0000147

29. Simon-Thomas ER, Keltner DJ, Sauter D, Sinicropi-Yao L, Abramson A. The voice conveys specific emotions: evidence from vocal burst displays. Emotion (2009) 9(6):838. doi: 10.1037/a0017810

30. Hoffner C, Badzinski DM. Children's integration of facial and situational cues to emotion. Child Dev (1989) 60(2):411-22. doi: 10.2307/1130986

31. Avinun R, Knafo A. The Longitudinal Israeli Study of Twins (LIST)-An integrative view of social development. Twin Res Hum Gen (2013) 16(1):197201. doi: $10.1017 /$ thg. 2012.73

32. Nowicki S, Duke MP. Manual for the receptive tests of the diagnostic analysis of nonverbal accuracy 2 (DANVA2). Atlanta, GA: Department of Psychology, Emory University (2008).

33. Rothman AD, Nowicki S. A measure of the ability to identify emotion in children's tone of voice. J Nonverbal Behav (2004) 28(2):67-92. doi: 10.1023/B:JONB.0000023653.13943.31

34. Guyer AE, McClure EB, Adler AD, Brotman MA, Rich BA, Kimes AS, et al. Specificity of facial expression labeling deficits in childhood psychopathology. J Child Psychol Psychiatry (2007) 48(9):863-71. doi:10.1111/j.1469-7610. 2007.01758.x

35. Sheaffer BL, Golden JA, Bridgers KT, Hall CW. Nonverbal processing and social competency in children with reactive attachment disorder. Behav Dev Bull (2009) 15(1):11-17. doi: 10.1037/h0100509

36. Walker AS, Nowicki S, Jones J, Heimann L. Errors in identifying and expressing emotion in facial expressions, voices, and postures unique to social anxiety. J Genet Psychol (2011) 172(3):293-301. doi: 10.1080/00221325.2010.535224

37. Neale MC, Boker SM, Xie G, Maes HM. Statistical modeling. Richmond, VA: Virginia Commonwealth University: Department of Psychiatry (1999).

38. Rijsdijk FV, Sham PC. Analytic approaches to twin data using structural equation models. Brief Bioinform (2002) 3(2):119-33. doi: 10.1093/bib/ 3.2.119

39. Ferretti V, Papaleo F. Understanding others: emotion recognition abilities in humans and other animals. Genes Brain Behav (2018) e12544:1-2. doi: $10.1111 /$ gbb. 12544

40. Elfenbein HA. Nonverbal dialects and accents in facial expressions of emotion. Emot Rev (2013) 5(1):90-6. doi: 10.1177/1754073912451332

41. Elfenbein HA. The many faces of emotional contagion: An affective process theory of affective linkage. Organ Psychol Rev (2014) 4(4):326-62. doi: $10.1177 / 2041386614542889$
42. Barrett LF. How emotions are made: The secret life of the brain. Houghton Mifflin Harcourt, Boston (2017).

43. Hetherington EM, Reiss D, Plomin R. Separate social worlds of siblings: The impact of nonshared environment on development. New York: Routledge (2013).

44. Davis MH, Luce C, Kraus SJ. The heritability of characteristics associated with dispositional empathy. J Pers (1994) 62(3):369-91. doi: 10.1111/j.14676494.1994.tb00302.x

45. Ronald A, Happe F, Hughes C, Plomin R. Nice and nasty theory of mind in preschool children: nature and nurture. Soc Dev (2005) 14(4):664-84. doi: 10.1111/j.1467-9507.2005.00323.x

46. Avinun R, Knafo A. Parenting as a reaction evoked by children's genotype: a meta-analysis of children-as-twins studies. Pers Soc Psychol Rev (2014) 18(1):87-102. doi: $10.1177 / 1088868313498308$

47. Haworth CM, Wright MJ, Luciano M, Martin NG, de Geus EJ, van Beijsterveldt CE, et al. The heritability of general cognitive ability increases linearly from childhood to young adulthood. Mol Psychiatry (2010) 15(11):1112. doi: 10.1038/mp.2009.55

48. Plomin R, Deary IJ. Genetics and intelligence differences: five special findings. Mol Psychiatry (2015) 20(1):98. doi: 10.1038/mp.2014.105

49. Knafo A, Zahn-Waxler C, Van Hulle C, Robinson J, Rhee SH. The developmental origins of a disposition towards empathy: genetic and environmental contributions. Emotion (2008) 8:737-52. doi: 10.1037/a0014179

50. Beam CR, Turkheimer E. Phenotype-environment correlations in longitudinal twin models. Dev Psychopathol (2013) 25(1):7-16. doi: 10.1017/ S0954579412000867

51. Scarr S, McCartney K. How people make their own environments: a theory of genotype $\rightarrow$ environment effects. Child Dev (1983) 54(2):424-35. doi: $10.2307 / 1129703$

52. Hasenfratz L, Benish-Weisman $M$, Steinberg $T$, Knafo-Noam A. Temperament and peer problems from early to middle childhood: geneenvironment correlations with negative emotionality and sociability. Dev Psychopathol (2015) 27:1089-109. doi: 10.1017/S095457941500070X

53. Nowicki S, Mitchell J. Accuracy in identifying affect in child and adult faces and voices and social competence in preschool children. Genet Soc Gen Psychol Monogr (1998) 124(1):39-60.

Conflict of Interest Statement: The authors declare that the research was conducted in the absence of any commercial or financial relationships that could be construed as a potential conflict of interest.

Copyright (๑ 2019 Schapira, Anger Elfenbein, Amichay-Setter, Zahn-Waxler and Knafo-Noam. This is an open-access article distributed under the terms of the Creative Commons Attribution License (CC BY). The use, distribution or reproduction in other forums is permitted, provided the original author(s) and the copyright owner(s) are credited and that the original publication in this journal is cited, in accordance with accepted academic practice. No use, distribution or reproduction is permitted which does not comply with these terms. 